\title{
The role of carotid chemoreceptors in the sympathetic activation by adenosine in humans
}

\author{
Henri J. L. M. TIMMERS*, Gerard A. RONGEN*, John M. KAREMAKER†, \\ Wouter WIELING $\ddagger$, Henri A. M. MARRES§ and Jacques W. M. LENDERS* \\ *Department of General Internal Medicine, University Medical Center Nijmegen, PO Box 910I, 6500 HB Nijmegen, The \\ Netherlands, †Department of Physiology, Academic Medical Center, P0 Box 22660, II00DD Amsterdam, The Netherlands, \\ $\ddagger$ Department of Internal Medicine, Academic Medical Center, PO Box 22660, IIO0DD Amsterdam, The Netherlands, and \\ §Department of Otorhinolaryngology, University Medical Center Nijmegen, PO Box 910I, 6500 HB Nijmegen, The Netherlands
}

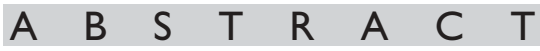

\begin{abstract}
The direct vasodilatory and negative chronotropic effects of adenosine in humans are counterbalanced by a reflex increase in sympathetic nerve traffic. A suggested mechanism for this reflex includes peripheral chemoreceptor activation. We, therefore, assessed the contribution of carotid chemoreceptors to sympatho-excitation by adenosine. Muscle sympathetic nerve activity was recorded during adenosine infusion $\left(140 \mu \mathrm{g} \cdot \mathrm{kg}^{-1} \cdot \mathrm{min}^{-1}\right.$ for $5 \mathrm{~min}$ ) in five patients lacking carotid chemoreceptors after bilateral carotid body tumour resection (one male and four female, mean age $5 \mathrm{I} \pm \mathrm{II}$ years) and in six healthy controls (two male and four female, mean age $50 \pm 7$ years). Sympathetic responses to sodium nitroprusside injections were assessed to measure baroreceptor-mediated sympathetic activation. In response to adenosine, controls showed no change in blood pressure, an increase in heart rate $(+48.2 \pm 13.2 \% ; P<0.003)$ and an increase in sympathetic nerve activity $(+195 \pm 103 \% ; P<0.022)$. In contrast, patients showed a decrease in blood pressure $(-14.6 \pm 4.9 /-17.6 \pm 6.0 \% ; P<0.05)$, an increase in heart rate $(+25.3 \pm 8.4 \%$; $P<0.032)$ and no significant change in sympathetic activity. Adenosine-induced hypotension in individual patients elicited less sympathetic activation than equihypotensive sodium nitroprusside injections. In humans lacking carotid chemoreceptors, adenosine infusion elicits hypotension due to the absence of significant sympatho-excitation. Chemoreceptor activation is essential for counterbalancing the direct vasodilation by adenosine. In addition, blunting of the baroreflex sympathetic response to adenosine-induced hypotension may indicate a direct sympatho-inhibitory effect of adenosine.
\end{abstract}

\section{INTRODUCTION}

The clinical use of adenosine as a therapeutic agent in supraventricular tachycardia [1] and as a diagnostic tool in adenosine-thallium stress testing for cardiac ischaemia [2] is based on its negative chrono- and dromo-tropic [3] and vasodilatory [4] properties respectively. However, in conscious healthy subjects, adenosine infusion results in a dose-related increase in heart rate (HR) and respiration without a change in mean arterial blood pressure (BP) [5-7]. Hypotension and bradycardia are prevented by an increase in sympathetic nerve activity in response to adenosine $[5,8]$. This sympatho-excitation reflex to vasodilation is responsible for the absence of hypotension and bradycardia, since, in patients with severe autonomic failure and in anaesthetized subjects, in whom

Key words: adenosine, carotid artery, chemoreceptor, chemoreflex, sympathetic nervous system.

Abbreviations: BP, blood pressure; BCBR, bilateral carotid body resection; DBP, diastolic BP; HR, heart rate; LBNP, lower body negative pressure; MSNA, muscle sympathetic nerve activity; SBP, systolic BP; SNP, sodium nitroprusside; TIA, total integrated activity.

Correspondence: Dr Henri J. L. M. Timmers (e-mail H.Timmers@aig.umcn.nl). 
Table I Characteristics of individual BCBR patients

CBR, carotid body tumour resection. *According to surgical and pathological reports. $\mathrm{nIX}$, ninth cranial nerve (glossopharyngeal nerve).

\begin{tabular}{|c|c|c|c|c|c|}
\hline Parameters & Patient I & Patient 2 & Patient 3 & Patient 4 & Patient 5 \\
\hline Age (years) & 44 & 58 & 44 & 62 & 36 \\
\hline Sex & Female & Female & Female & Female & Male \\
\hline \multicolumn{6}{|l|}{ First CBR } \\
\hline Time between surgery and investigation (years) & 7.1 & 20.4 & 7.8 & 5.3 & 7.4 \\
\hline Side & Right & Left & Left & Left & Left \\
\hline Tumour size $(\max . \mathrm{cm})^{*}$ & 2.5 & 2.0 & 4.0 & 3.0 & 2.0 \\
\hline Radical resection* & Yes & No & Yes & Yes & Yes \\
\hline (Cranial) nerve damage* & - & - & $\mathrm{nIX}$ & - & - \\
\hline \multicolumn{6}{|l|}{ Second CBR } \\
\hline Time between surgery and investigation (years) & 6.1 & 20.3 & 6.7 & 4.4 & 6.6 \\
\hline Side & Left & Right & Right & Right & Right \\
\hline Tumour size $(\max . \mathrm{cm})^{*}$ & 1.0 & 1.5 & 3.0 & 2.0 & 1.5 \\
\hline Radical resection* & Yes & No & Yes & No & Yes \\
\hline (Cranial) nerve damage* & - & Cervical plexus & - & - & - \\
\hline Symptoms/signs of baroreflex failure & Persistent & - & - & - & Transient \\
\hline Additional paraganglioma localizations/therapy & - & $\begin{array}{l}\text { Right side skull basis/ } \\
\text { radiotherapy }\end{array}$ & - & - & - \\
\hline Family history of paraganglioma & Yes & Yes & No & Yes & Yes \\
\hline Medication & $\begin{array}{l}\text { Chloorthalidon, atenolol } \\
\text { and moxonidine }\end{array}$ & - & - & Atenolol & - \\
\hline
\end{tabular}

autonomic reflexes are blunted, adenosine produces sustained hypotension [9,10].

Mechanisms that have been suggested to contribute to a reflex increase in muscle sympathetic nerve activity (MSNA) include activation of peripheral chemoreceptors [7], unloading of arterial and cardiopulmonary baroreceptors $[8,11]$ and stimulation of adenosine-sensitive afferent vessels in the heart [12], kidney [13] and forearm [14]. Adenosine-induced activation of peripheral chemoreceptors has been demonstrated in animal studies $[15,16]$. Indirect evidence for the involvement of peripheral chemoreceptors in the sympathetic activation by adenosine in humans was provided by a differential effect of adenosine on BP, which was dependent on the site of infusion. Adenosine (with a very short half-life of $<1.5 \mathrm{~s}$ [17]) has pressor effects when administered in the aortic arch proximal to the origin of the carotid arteries, but depressor effects when the carotid body chemoreceptors are by-passed by administration at the level of the descending aorta [7,18]. Furthermore, the MSNA and HR responses to adenosine are blunted when peripheral chemoreceptors are silenced by hyperoxia [11]. However, the relative impact of chemoreceptor activation on sympatho-excitation by adenosine in humans remains to be elucidated.

In a previous study [19], we have demonstrated that carotid body chemoreflex function is abolished in patients after removal of a bilateral carotid paraganglioma [bilateral carotid body resection (BCBR)], as indicated by the absence of a ventilatory response to normocapnic hypoxaemia in these patients. The aim of the present study was to examine the role of peripheral chemoreceptors in the sympathetic activation by exogenous adenosine by comparing MSNA responses to continuous intravenous infusion of adenosine between BCBR patients (lacking carotid chemoreceptors) and healthy controls. In addition, MSNA responses to sodium nitroprusside (SNP) injections were assessed for estimation of baroreceptor-mediated sympathetic activation.

\section{METHODS}

\section{Patients and controls}

Five patients (one male and four females), who had undergone BCBR at the Department of Otolaryngology, University Medical Center Nijmegen, The Netherlands, were included in this study. Individual information on tumour size, additional tumour localizations and surgical details of these five patients are shown in Table 1. The median interval between the second operation and the study was 6.7 years (range, 4.4-20.3 years). Patients were free of diabetes, neurological, cardiovascular and pulmonary disease. Six healthy subjects (two males and four females) served as controls. Full medical history and physical examination, including BP measurements, revealed no abnormalities. Groups were matched for age $(51.2 \pm 10.8$ years in BCBR patients compared with 
$50.0 \pm 6.5$ years in controls), body mass index $(24.8 \pm$ $1.1 \mathrm{~kg} / \mathrm{m}^{2}$ in patients compared with $25.7 \pm 3.8 \mathrm{~kg} / \mathrm{m}^{2}$ in controls) and alcohol intake (7.8 \pm 8.5 units/week in patients compared with $9.0 \pm 7.4$ units/week in controls). The study protocol was carried out in accordance with the Declaration of Helsinki (2000) of the World Medical Association and was approved by the Institutional Ethics Committee. All subjects gave their informed consent. The procedures followed were in accordance with Institutional guidelines.

\section{$B P, H R$ and breathing frequency}

Investigations were carried out during the morning after an overnight fast in a room with an ambient temperature of $22-24^{\circ} \mathrm{C}$. Subjects abstained from caffeine, tea, alcohol, chocolate and smoking for at least $24 \mathrm{~h}$ [20]. Office systolic and diastolic BP (SBP/DBP) was determined from the mean of three supine sphygmomanometric measurements after $10 \mathrm{~min}$ of rest. Continuous finger arterial BP was monitored by a Finapres device (model 5) [21], and HR by surface ECG connected to a Hewlett Packard 378341A monitor. Respiratory rate was monitored by changes in inspiratory and expiratory air temperature by means of a nose thermistor (Fysicon Medical Technology). An intravenous line was placed in an anticubital vein for adenosine infusion.

\section{Sympathetic nerve recordings}

Multi-unit microneurographic recordings of postganglionic MSNA were obtained with a unipolar tungsten electrode inserted selectively into a muscle-nerve fascicle of the right peroneal nerve posterior to the fibula head, as originally described by Sundlöf and Wallin [22]. A reference electrode was inserted subcutaneously $1-3 \mathrm{~cm}$ from the recording electrode. Electrodes were connected to a preamplifier with a gain of 1000 and an amplifier with a gain that could be varied from $30-90$ as required in each subject. Amplification was constant throughout the study in each subject. Neural activity was fed through a bandpass filter with a bandwidth of $700-2000 \mathrm{~Hz}$. The filtered neurogram was routed through an amplitude discriminator to a storage oscilloscope and a loudspeaker. For recording and analysis, the filtered neurogram was fed through a resistance-capacitance integrating network (time constant $0.1 \mathrm{~s}$ ) to obtain a mean voltage neurogram of MSNA. Acceptable recordings met the following criteria: spontaneous bursts of neural discharge synchronous with HR, no response to arousal stimuli or skin stroking and an increase in nerve burst frequency with apnoea.

\section{Study protocol}

After instrumentation, subjects lay quietly for a period of stabilization of $20 \mathrm{~min}$. After a 5-10 min baseline recording, adenosine (adenocor ${ }^{\circledR} ; 6 \mathrm{mg}$ in $2 \mathrm{ml}$ ) was administered intravenously at a dosage of $140 \mu \mathrm{g} \cdot \mathrm{kg}^{-1}$. $\mathrm{min}^{-1}$ during a 5 min period. This infusion rate is similar to the dose that is applied during diagnostic myocardial stress testing [23].

To assess the baroreflex control of sympathetic outflow, we calculated the increase in MSNA in response to a decrease in BP induced by SNP [24]. After a $20 \mathrm{~min}$ baseline period, graded bolus injections of SNP (12.5, 25,50 and $100 \mu \mathrm{g}$ ) were given intravenously. The dosage producing a decrease in arterial pressure of $\approx 15 \mathrm{mmHg}$ was repeated three times at intervals of $10 \mathrm{~min}$.

\section{Data analysis}

A computer-assisted method was applied for automatic detection and quantification of individual bursts of sympathetic nerve activity by means of a curve-fitting method. During a prescan of the tracing, the neurogram was correlated with a triangular signal by applying a least-squares algorithm. In order to define 'reference bursts', the 100 largest triangular waves detected during the prescan were taken. After discarding the 20 largest waves for possible artefacts, the remaining 80 were taken as a reference. Their mean amplitude and delay from the corresponding R-wave on the ECG were determined. During a subsequent scan, the individual amplitude and time delay of all triangular waves were compared with the mean amplitude and delay of the reference waves. Waves were accepted and marked as sympathetic bursts on two conditions: an amplitude of $>20 \%$ of the mean reference amplitude and a time delay of $<200 \mathrm{~ms}$ beneath or above the mean reference delay. Automatically calculated burst amplitude correlated well $(r>0.9)$ with manual burst detection during supine rest and adenosine infusion (H. J. L. M. Timmers, G. A. Rongen and J. W. M. Lenders, unpublished work). MSNA was expressed as bursts $/ \mathrm{min}$, bursts $/ 100$ beats, total integrated activity (TIA)/min, TIA/100 beats and mean integrated activity/burst.

Responses to adenosine infusion were expressed as relative changes in BP, HR, breathing frequency and MSNA, calculated as $1 \mathrm{~min}$ averages during the $5 \mathrm{~min}$ of adenosine infusion and 5 min of recovery compared with $1 \mathrm{~min}$ at baseline.

The relative increase in MSNA during a $20 \mathrm{~s}$ stable BP minimum after SNP injection was taken as an estimate of baroreflex control of sympathetic outflow during sustained hypotension. In subjects that exhibited a hypotension in response to adenosine, the relative increase in MSNA was compared with the increase in MSNA elicited by an equihypotensive dose of SNP in that particular patient. For comparison of MSNA responses to adenosine- and SNP-induced hypotension, we selected a $1 \mathrm{~min}$ interval during adenosine infusion in which the decrease in DBP was equal to the mean SNP-induced decrease in BP. The MSNA response to SNP was taken as the expected baroreflex-mediated sympathetic response to hypotension during adenosine infusion [8]. 


\section{Statistics}

Results are given as means \pm S.E.M. unless indicated otherwise. Differences between patients and controls with respect to nominal variables were compared using the $\chi^{2}$ test. Other variables were compared using Student's $t$ test or Mann-Whitney rank-sum test when appropriate. A two-sided $P<0.05$ was taken as the level of significance. Statistical analysis was performed using SPSS for Windows 6.1.3.

\section{RESULTS}

\section{Sympathetic response(s) to adenosine}

At baseline, sphygmomanometric SBP/DBP and HR did not differ between groups (120.8 $\pm 11.1 / 79.0 \pm$ $5.9 \mathrm{mmHg}$ and $66.8 \pm 8.5 \mathrm{bpm}$ in patients compared with $123.3 \pm 11.9 / 81.5 \pm 7.6 \mathrm{mmHg}$ and $66.2 \pm 6.5 \mathrm{bpm}$ in controls) and neither did sympathetic burst frequency (39.6 \pm 20.0 bursts/min and $49.3 \pm 21.5$ bursts $/ 100$ beats in patients compared with $38.8 \pm 11.5$ bursts $/ \mathrm{min}, 59.0 \pm$ 19.8 bursts $/ 100$ beats in controls).

Patients and controls retrospectively reported the following symptoms during adenosine infusion: chest pain (all patients and controls), dyspnoea (patients 1-3, controls 1-4 and 6), flushing (patient 1), headache (patient 1), nausea (patient 4 and control 5) and nervousness (patient 1).

Representative tracings of BP, HR and MSNA responses to adenosine, as observed in control 4 and BCBR patient 4, are depicted in Figure 1. Group-averaged responses during the fifth minute of adenosine infusion compared with baseline are shown in Figure 2. Controls had no significant change in SBP/DBP $(+1.1 \pm 2.6 /$ $-2.1 \pm 2.0 \%)$, but significant increases in HR (48.2 \pm $13.2 \% ; P<0.003)$, MSNA expressed as TIA $/ \mathrm{min}$ $(195 \pm 103 \% ; P<0.022)$ and integrated activity/burst $(38.0 \pm 12.0 \% ; P<0.036)$. The increase in MSNA expressed as bursts/min ( $+96 \pm 48 \%)$, bursts $/ 100$ beats $(+39.6 \pm 38 \%)$ and TIA/100 beats $(+113 \pm 79 \%)$ in controls were not significant. BCBR patients showed a significant decrease in SBP/DBP of $-14.6 \pm 4.9$ / $-17.6 \pm 6.0 \% \quad(P<0.05)$ and a significant increase in HR of $+25.3 \pm 8.4 \%(P<0.032)$, and non-significant increases in MSNA of $+63.1 \pm 30.8 \% \mathrm{TIA} / \mathrm{min},+7.4 \pm$ $7.8 \%$ mean integrated activity/burst, $+50.6 \pm 27.5 \%$ bursts $/ \mathrm{min}, \quad+18.2 \pm 16.8 \%$ bursts $/ 100$ beats and $+27.5 \pm 19.3 \%$ TIA/100 beats. The responses differed significantly between patients and controls with respect to SBP $(P<0.04)$, DBP $(P<0.004)$ and mean amplitude/ burst $(P<0.021)$. There was a trend towards a smaller increase in $\mathrm{HR}$ in patients compared with controls $(P<0.094)$. After the infusion, changes in BP, HR and MSNA returned to baseline in both groups within 35 min. Mean respiratory rates during adenosine infusion were not different from baseline in patients $(14.4 \pm 4.4$
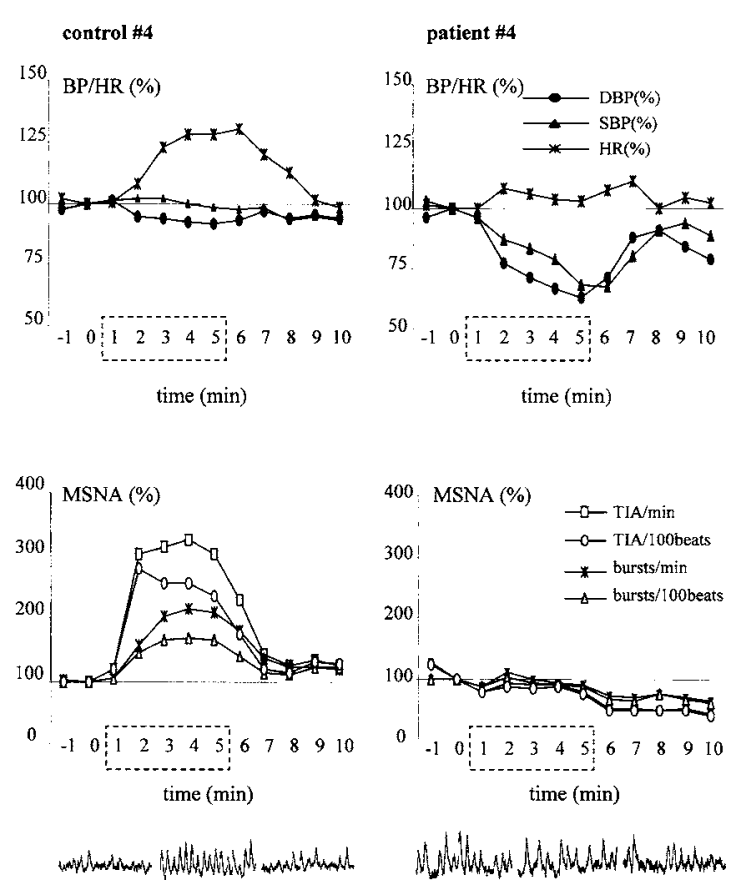

Figure I Individual responses to adenosine infusion in a representative control (left panel) and patient (right panel) The data are presented as I-min-averaged relative changes in DBP, SBP, HR and MSNA (TIA) during (I-5 min, broken-lined box) and after (6-10 min) adenosine infusion compared with baseline (-I-O min). The neurogram of the last $10 \mathrm{~s}$ before, the fifth minute during and the fifth min after infusion are displayed at the bottom.

and $16.4 \pm 1.9 / \mathrm{min}$ respectively) or controls (13.8 \pm 2.6 and $14.3 \pm 2.2 / \mathrm{min}$ respectively).

\section{Sympathetic response(s) to SNP}

MSNA responses to SNP-induced hypotension compared with changes in MSNA in the four BCBR patients that exhibited a BP decrease during adenosine infusion (patients 2-5) are shown in Figure 3. Adenosine elicited smaller increases in MSNA than equihypotensive doses of SNP.

\section{DISCUSSION}

In the present study, we have investigated the role of peripheral chemoreceptors in the sympathetic activation by exogenous adenosine by comparing MSNA responses to continuous intravenous infusion of adenosine $(140 \mu \mathrm{g}$. $\mathrm{kg}^{-1} \cdot \min ^{-1}$ ) between BCBR patients, lacking carotid chemoreceptors, and healthy controls. Our principle finding is that absence of carotid body chemoreflex function due to BCBR results in abolishment of significant MSNA and HR responses to adenosine. In the absence of chemoreceptor-dependent sympatho-excitation, adenosine infusion causes a $15 \%$ decrease in BP. Furthermore, 

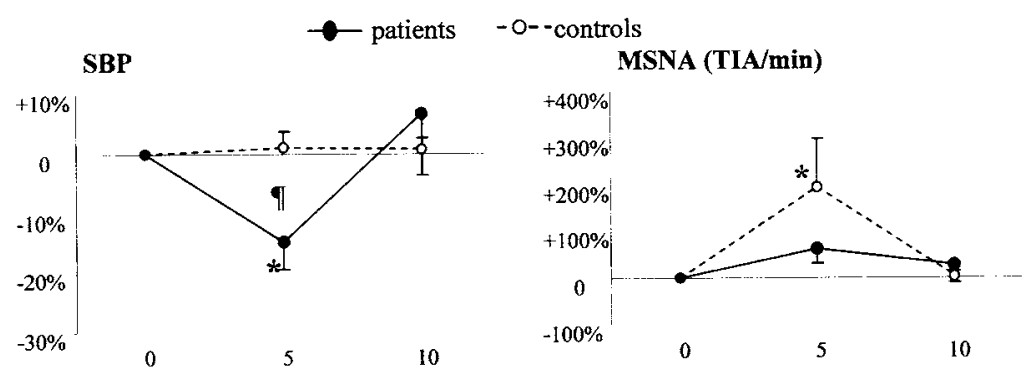

DBP
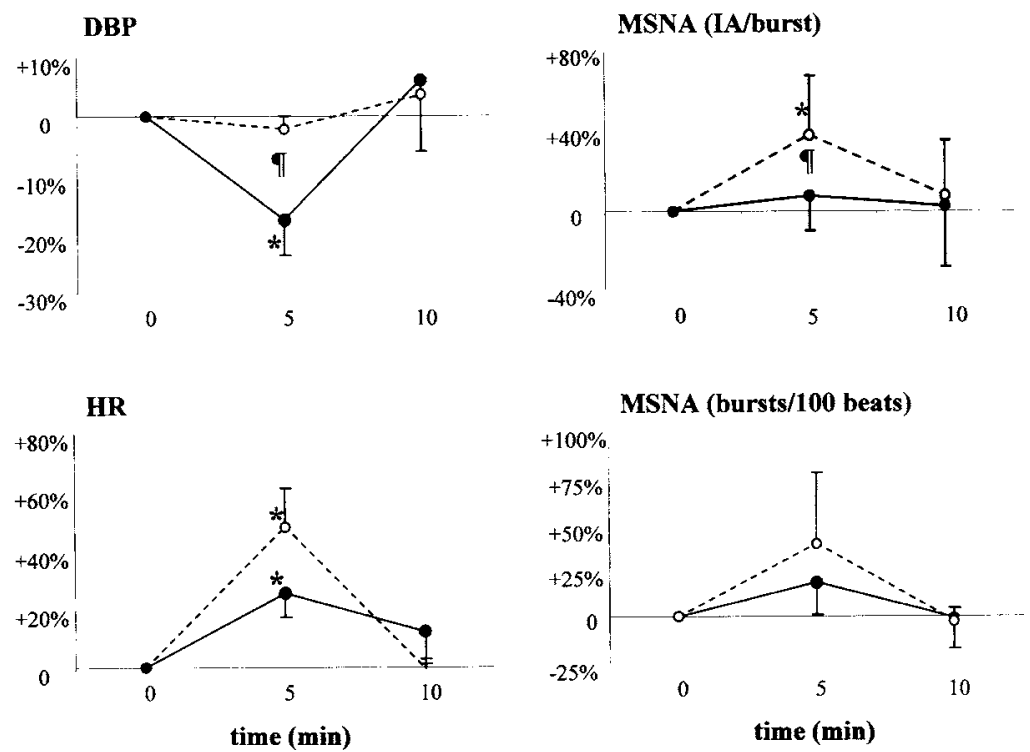

Figure 2 Group-averaged responses to adenosine in patients and controls

Data are presented as relative mean \pm S.E.M. changes from baseline in SBP, DBP, HR and MSNA (TIA) during the last min of adenosine infusion $(t=5 \mathrm{~min})$ and after return to baseline $(t=10 \mathrm{~min})$. "Significant within-group change from baseline $(P<0.05)$, Tsignificant difference in fifth min responses between controls and patients.

the BP decrease induced by adenosine in BCBR patients elicited a smaller increase in MSNA than expected from the observed individual baroreflex-mediated MSNA responses to SNP.

It has been well established [5-8], that continuous adenosine infusion in healthy awake humans causes a dose-dependent increase in HR, MSNA and plasma noradrenaline levels without a change in mean arterial BP. The tachycardic response correlates significantly with increases in noradrenaline [5]. In a previous study [25], spectral analysis of HR showed a reduced parasympathetic and increased sympathetic influence on the sinus node during intravenous infusion of adenosine in healthy volunteers. Our present observations of blunted MSNA and HR responses and hypotension in response to adenosine infusion in patients lacking carotid chemoreceptors indicate that carotid chemoreceptors are indispensable in triggering autonomic reflexes during adenosine infusion, which prevent hypotension that would otherwise occur because of the direct vasodilatory action of adenosine [26]. Evidence for peripheral chemoreceptor activation by adenosine comes from animal studies in which intracarotid infusion of adenosine causes an $\mathrm{A}_{2}$-receptor-specific stimulation of afferent chemoreceptor nerve traffic and ventilation $[15,16]$. In humans, intravenous adenosine infusion produces a dose-related increase in ventilation, due mostly to increased depth of respiration $[5,6]$ even at a dose below the threshold for symptoms $[7,27]$. In line with these observations, we found no effect of adenosine on respiratory rate in either patients or controls. For practical reasons, we did not perform spirometry during the infusion, so tidal volume was not measured. Respiratory stimulation by adenosine is suggested to result from peripheral chemoreflex activation, as ventilatory stimulation only occurs when adenosine is injected proximal to the origin of carotid arteries (and carotid chemoreceptors) and not after administration in the descending aorta $[7,18]$. The finding that adenosine increases the ventilatory response to hypoxia, not to hypercapnia, reiterates its stimulatory effect on peripheral chemoreceptors [27]. Previous studies $[7,8,11,18]$ on the role of peripheral chemoreceptor activation in mechanism of sympatho-excitation by adenosine infusion in humans have yielded merely indirect 


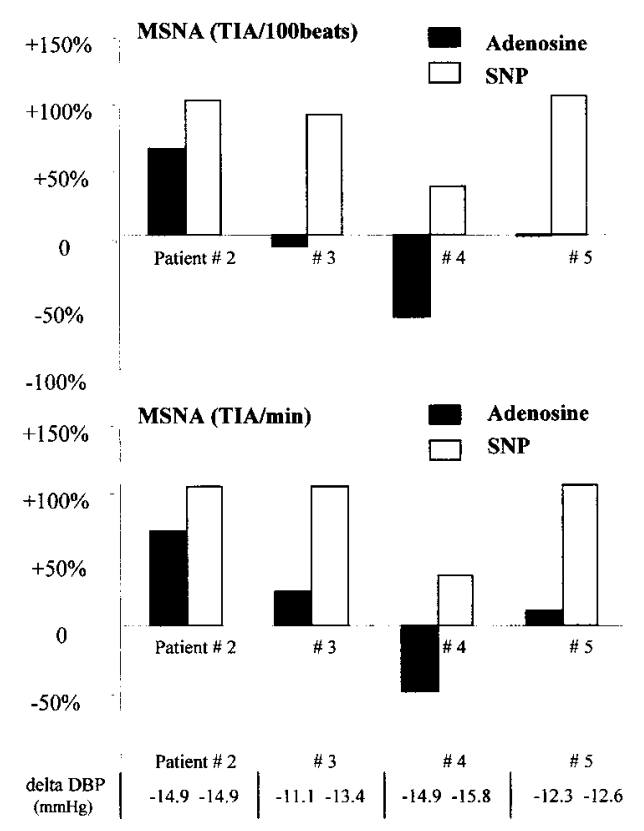

Figure 3 Changes in MSNA in response to equihypotensive doses of adenosine (closed bars) and SNP (open bars) in individual patients

Relative changes in TIA/I00 beats (upper panel) and TIA/min (lower panel) in response to changes in DBP induced by adenosine and SNP. It should be noted that, within each patient, adenosine and SNP induce similar decreases in DBP (indicated at the bottom of the Figure) and that similar BP reductions elicit smaller MSNA responses during adenosine infusion than during SNP infusion.

evidence. Adenosine was shown $[7,18]$ to have a differential pressor and depressor effect depending on infusion proximal or distal to the carotid chemoreceptors respectively. In addition, suppression of peripheral chemoreceptors by hyperoxia was shown to decrease MSNA and HR response to adenosine by one-third compared with the normoxic response [11], and adenosine infusion causes HR and MSNA responses similar to that observed during an equal ventilatory stimulation by hypoxia [8].

We have reported previously [28] that BCBR has a profound effect on peripheral chemoreflex function, as suggested by the complete abolishment of the normocapnic ventilatory responsiveness to hypoxia. During simultaneous hypercapnia, a small hypoxic response was observed in two of eight patients (patients 4 and 5 in the present study). This residual chemoreflex function was attributed to the remaining aortic bodies. These subserve a minor role in the modulation of spontaneous respiratory activity in humans, but they may generate a discernible response when their gain is increased by hypercapnia [29]. Apart from carotid body chemoreceptor stimulation, adenosine was shown to increase aortic chemoreceptor discharge in cats [30]. However, blunted MSNA responses in BCBR patients suggest little, if any, contri- bution of aortic chemoreceptors to the sympathoexcitation by adenosine.

Other possible mechanisms for sympatho-excitation by adenosine in humans, besides chemoreceptor activation, include stimulation of adenosine-sensitive afferent vessels in the coronary arteries [12], kidney [13] and forearm [14]. In addition, a reflex increase in MSNA may be triggered by unloading of the arterial and cardiopulmonary baroreceptors by the direct vasodilator action of adenosine $[8,11]$. In healthy subjects, adenosine was shown to produce a greater increase in MSNA than SNP injections at doses that resulted in equivalent hypotension [8]. Arterial baroreceptor unloading, therefore, does not completely explain the increase in sympathetic traffic produced by adenosine. On the other hand, mechanical baroreceptor unloading by $-15 \mathrm{mmHg}$ lower body negative pressure (LBNP) elicited an MSNA response similar to that observed during an equihypotensive dose of adenosine [11]. However, this level of LBNP causes both arterial and cardiopulmonary baroreceptor unloading, and the impact of LBNP and adenosine on central venous pressure (and thereby on cardiopulmonary mechano-receptors) were not compared. Adenosine was reported to cause no change [8], or even a small increase [31], in central venous pressure, which makes unloading of cardiopulmonary receptors unlikely. Furthermore, caffeine abstinence was less well implemented in the LBNP study [11], which may have underestimated the autonomic response to adenosine [20].

In a previous study [28], we have shown that BCBR results in attenuation of the baroreflex control of HR. The decrease in baroreflex sensitivity after BCBR was suggested to be due to partial denervation of the carotid sinus baroreceptors. However, SNP-induced hypotension in these patients elicited a prominent baroreceptormediated rise in MSNA, which was even larger than the MSNA response to an equivalent decrease in BP during adenosine infusion. We speculate that this finding is explained by an inhibitory role of adenosine on ganglionic neurotransmission [32]. Direct central depression of sympathetic tone by exogenous adenosine [33] is unlikely to occur, since adenosine crosses the blood-brain barrier very slowly [34]. On the other hand, the sympathetic nuclei lie close to the area postrema, which was shown to be permeable to adenosine in animal studies [35]. In healthy awake individuals, this direct sympatholytic effect of adenosine might be overwhelmed by the reflex activation of sympathetic nerve traffic.

The findings of hypotension and absence of a significant MSNA increase during adenosine infusion in BCBR patients compared with the presence of a baroreflex-mediated MSNA response to SNP suggests that neither baroreceptor unloading nor stimulation of adenosine-sensitive afferents, other than carotid body chemoreceptors, contribute significantly to the mechanism of sympatho-excitation by intravenous adenosine. 
Interruption of the chemoreflex influence on sympathetic outflow and unopposed direct vasodilatory action probably also accounts for adenosine-induced hypotension during general anaesthesia [9]. The finding of an incidence of hypotension (decrease in SBP $\geqslant-30 \mathrm{mmHg}$ ) of $10 \%$ (116 out of 1000) in patients undergoing adenosinethallium stress testing for cardiac ischaemia may be due to the large prevalence of diabetes $(25 \%)$ among these patients [23]. Diabetic autonomic neuropathy may cause a disruption of the efferent sympathetic pathway by which adenosine brings about reflex vasoconstriction. This may also explain adenosine-induced hypotension in patients with severe autonomic failure [7].

\section{Conclusions}

The present study indicates that carotid chemoreceptors play a key role in the sympatho-excitation during intravenous adenosine infusion in humans. This chemoreceptor-dependent increase in MSNA prevents adenosine-induced hypotension by counterbalancing its direct vasodilatory action. In addition, blunting of the baroreflex-mediated MSNA response to adenosineinduced hypotension may indicate a direct sympathoinhibitory effect of adenosine.

\section{ACKNOWLEDGMENTS}

This project was supported by a grant from The Netherlands Heart Foundation.

\section{REFERENCES}

1 DiMarco, J. P., Sellers, T. D., Berne, R. M., West, G. A. and Belardinelli, L. (1983) Adenosine: electrophysiologic effects and therapeutic use for terminating paroxysmal supraventricular tachycardia. Circulation 68, 1254-1263

2 Cerqueira, M. D., Verani, M. S., Schwaiger, M., Heo, J. and Iskandrian, A. S. (1994) Safety profile of adenosine stress perfusion imaging: results from the Adenoscan Multicenter Trial Registry. J. Am. Coll. Cardiol. 23, 384-389

3 Belhassen, B. and Pelleg, A. (1984) Acute management of paroxysmal supraventricular tachycardia: verapamil, adenosine triphosphate or adenosine? Am. J. Cardiol. 54, 225-227

4 Berne, R. M. (1980) The role of adenosine in the regulation of coronary blood flow. Circ. Res. 47, 807-813

5 Biaggioni, I., Onrot, J., Hollister, A. S. and Robertson, D. (1986) Cardiovascular effects of adenosine infusion in man and their modulation by dipyridamole. Life Sci. 39, 2229-2236

6 Watt, A. H. and Routledge, P. A. (1985) Adenosine stimulates respiration in man. Br. J. Clin. Pharmacol. 20, 503-506

7 Biaggioni, I., Olafsson, B., Robertson, R. M., Hollister, A. S. and Robertson, D. (1987) Cardiovascular and respiratory effects of adenosine in conscious man. Evidence for chemoreceptor activation. Circ. Res. 61, 779-786

8 Biaggioni, I., Killian, T. J., Mosqueda-Garcia, R., Robertson, R. M. and Robertson, D. (1991) Adenosine increases sympathetic nerve traffic in humans. Circulation 83, 1668-1675
9 Sollevi, A., Lagerkranser, M., Irestedt, L., Gordon, E. and Lindquist, C. (1984) Controlled hypotension with adenosine in cerebral aneurysm surgery. Anesthesiology 61, 400-405

10 Zimpfer, M., Sit, S. P. and Vatner, S. F. (1981) Effects of anesthesia on the canine carotid chemoreceptor reflex. Circ. Res. 48, 400-406

11 Rongen, G. A., Senn, B. L., Ando, S., Notarius, C. F., Stone, J. A. and Floras, J. S. (1997) Comparison of hemodynamic and sympathoneural responses to adenosine and lower body negative pressure in man. Can. J. Physiol. Pharmacol. 75, 128-134

12 Cox, D. A., Vita, J. A., Treasure, C. B., Fish, R. D., Selwyn, A. P. and Ganz, P. (1989) Reflex increase in blood pressure during the intracoronary administration of adenosine in man. J. Clin. Invest. 84, 592-596

13 Katholi, R. E., Whitlow, P. L., Hageman, G. R. and Woods, W. T. (1984) Intrarenal adenosine produces hypertension by activating the sympathetic nervous system via the renal nerves in the dog. J. Hypertens. 2, 349-359

14 Costa, F. and Biaggioni, I. (1993) Adenosine activates afferent fibers in the forearm, producing sympathetic stimulation in humans. J. Pharmacol. Exp. Ther. 267, 1369-1374

15 McQueen, D. S. and Ribeiro, J. A. (1986) Pharmacological characterization of the receptor involved in chemoexcitation induced by adenosine. Br. J. Pharmacol. 88, 615-620

16 Monteiro, E. C. and Ribeiro, J. A. (1987) Ventilatory effects of adenosine mediated by carotid body chemoreceptors in the rat. Naunyn-Schmiedebergs Arch. Pharmacol. 335, 143-148

17 Moser, G. H., Schrader, J. and Deussen, A. (1989) Turnover of adenosine in plasma of human and dog blood. Am. J. Physiol 256, C799-C806

18 Watt, A. H., Reid, P. G., Stephens, M. R. and Routledge, P. A. (1987) Adenosine-induced respiratory stimulation in man depends on site of infusion. Evidence for an action on the carotid body? Br. J. Clin. Pharmacol. 23, 486-490

19 Timmers, H. J., Karemaker, J. M., Lenders, J. W. and Wieling, W. (1999) Baroreflex failure following radiation therapy for nasopharyngeal carcinoma. Clin. Auton. Res. 9, 317-324

20 Rongen, G. A., Brooks, S. C., Ando, S., Notarius, C. F. and Floras, J. S. (1998) Caffeine abstinence augments the systolic blood pressure response to adenosine in humans. Am. J. Cardiol. 81, 1382-1385

21 Wesseling, K. H. (1996) Finger arterial pressure measurement with Finapres. Z. Kardiol. 85 (Suppl. 3), 38-44

22 Sundlöf, G. and Wallin, B. G. (1977) The variability of muscle nerve sympathetic activity in resting recumbent man. J. Physiol. (Cambridge, U.K.) 272, 383-397

23 Johnston, D. L., Daley, J. R., Hodge, D. O., Hopfenspirger, M. R. and Gibbons, R. J. (1995) Hemodynamic responses and adverse effects associated with adenosine and dipyridamole pharmacologic stress testing: a comparison in 2,000 patients. Mayo Clin. Proc. 70, 331-336

24 Ebert, T. J. and Cowley, Jr, A. W (1992) Baroreflex modulation of sympathetic outflow during physiological increases of vasopressin in humans. Am. J. Physiol 262, H1372-H1378

25 Rongen, G. A., Brooks, S. C., Pollard, M. J. et al. (1999) Effect of adenosine on heart rate variability in humans. Clin. Sci. 96, 597-604

26 Smits, P., Williams, S. B., Lipson, D. E., Banitt, P., Rongen, G. A. and Creager, M. A. (1995) Endothelial release of nitric oxide contributes to the vasodilator effect of adenosine in humans. Circulation 92, 2135-2141

27 Maxwell, D. L., Fuller, R. W., Nolop, K. B., Dixon, C. M. and Hughes, J. M. (1986) Effects of adenosine on ventilatory responses to hypoxia and hypercapnia in humans. J. Appl. Physiol 61, 1762-1766

28 Timmers, H. J. L. M., Karemaker, J. M., Wieling, W., Marres, H. A., Folgering, H. Th. M. and Lenders, J. W. M. (2003) Baroreflex and chemoreflex function after bilateral carotid body tumor resection. J. Hypertens. 21, 591-599 
29 Whipp, B. J. (1994) Carotid bodies and breathing in humans. Thorax 49, 1081-1084

30 Runold, M., Cherniack, N. S. and Prabhakar, N. R. (1990) Effect of adenosine on chemosensory activity of the cat aortic body. Respir. Physiol. 80, 299-306

31 Nussbacher, A., Arie, S., Kalil, R. et al. (1995) Mechanism of adenosine-induced elevation of pulmonary capillary wedge pressure in humans. Circulation 92, 371-379

32 Delle, M., Ricksten, S. E. and Delbro, D. (1988) Pre- and post-ganglionic sympathetic nerve activity during induced hypotension with adenosine or sodium nitroprusside in the anesthetized rat. Anesth. Analg. 67, 307-312
33 Tseng, C. J., Biaggioni, I., Appalsamy, M. and Robertson, D. (1988) Purinergic receptors in the brainstem mediate hypotension and bradycardia. Hypertension 11, 191-197

34 Berne, R. M., Rubio, R. and Curnish, R. R. (1974) Release of adenosine from ischemic brain: effect of cerebral vascular resistance and incorporation into cerebral adenosine nucleotides. Circ. Res. 35, 262-271

35 Faraci, F. M., Choi, J., Baumbach, G. L., Mayhan, W. G. and Heistad, D. D. (1989) Microcirculation of the area postrema. Permeability and vascular responses. Circ. Res. 65, 417-425

Received I 3 May 2003/29 July 2003; accepted 22 August 2003

Published as Immediate Publication 22 August 2003, DOI 10.1042/CS20030I74 